\title{
Características de la tuberculosis en pacientes mayores de 65 años en el área sanitaria de Cádiz (España)
}

\author{
Irache López-Pelayo ${ }^{1}$, Pedro G arcía-Martos ${ }^{1}$, \\ Abel Saldarreaga ${ }^{1}$, M ontserrat Montes de $0 \mathrm{ca}^{2}$, \\ Isabel Moreno ${ }^{3}$, Enrique G onzález-Moya ${ }^{4}$ \\ Characteristics of tuberculosis in \\ patients older than 65 years in the \\ sanitary area of Cadiz (Spain)
}

\begin{abstract}
Background: Tuberculosis in the elderly is an increasing public health problem. Aim: To describe the epidemiological, clinical and microbiological characteristics of tuberculosis in patients over 65 years old, as compared to younger patients. Material and methods: We reviewed the case histories of tuberculosis, microbiologically diagnosed by ZiehlNeelsen/auramine smear and Löwenstein-Jensen culture, in patients over 65 years old, in the sanitary area of Cadiz (Spain). Results: We detected 49 new cases (37 males) of tuberculosis in elderly subjects. The infection rate in this age group (16.3/100.000 inhabitants) is lower than in people younger than 65 years old (21.1/100.000 inhabitants). The most common symptoms were cough (44.9\%), fever (40.8\%) and dyspnea (44.9\%). Neither of them were infected by the human immunodeficiency virus nor were parenteral drug abusers. Thirty seven percent of cases had a history of previous tuberculosis, and in $75.5 \%$ the disease location was pulmonary. The ZiehlNeelsen/auramine smear was negative in $53.1 \%$ of the samples. Conclusions: The number of patients older than 65 years old with tuberculosis has increased in the sanitary area of Cadiz (Spain). This group of patients shows different epidemiological and clinical characteristics than younger patients (Rev Méd Chile 2004; 132: 325-30).
\end{abstract}

(Key Words: Aged; Epidemiologic studies; Tuberculosis)

Recibido el 23 de mayo, 2003. Aceptado en versión corregida el 25 de noviembre, 2003. Servicios de ${ }^{1}$ Microbiología, ${ }^{2}$ Medicina Interna, ${ }^{3}$ Medicina Familiar y ${ }^{4}$ Neumología, Hospital Universitario Puerta del Mar, Cádiz, España.

Correspondencia a: Pedro García-Martos. Servicio de Microbiología, Hospital Universitario Puerta del Mar. Ana de Viya, 21. 11009 Cádiz (España). Fono: 956-003068. Fax: 956-003081. E-mail: pedromartos@hotmail.com 
$E^{n}$ n los países desarrollados ha habido una disminución constante de la incidencia de tuberculosis hasta 1985, lo que hizo pensar en una posible erradicación de la enfermedad, pero al principio de la década 1990-99, se observó en España1-3 y en otros países $^{5,6}$ un aumento del número de casos debido, entre otras causas, a la infección por el virus de la inmunodeficiencia humana (VIH) y a la inmigración ${ }^{7}$. Por otra parte, también se ha detectado un aumento de las formas extrapulmonares y diseminadas y un fenómeno nuevo, la aparición de cepas resistentes a los tuberculostáticos de uso habitual ${ }^{7-11}$.

El grupo de personas mayores de 65 años constituye una parte de la población cada vez más numerosa en nuestro país. En ellos suele observarse un incremento de la incidencia de tuberculosis en relación con la población general, al presentar un mayor número de factores predisponentes, principalmente enfermedad pulmonar obstructiva crónica y neoplasias ${ }^{1-3}$. Esta población podría posiblemente contribuir de algún modo a la alta persistencia de tuberculosis en España ${ }^{1-4}$.

El objetivo de nuestro trabajo ha sido estudiar la evolución de la tuberculosis en pacientes mayores de 65 años en el área sanitaria de Cádiz (España), desde 1997 hasta 2002, y describir las características epidemiológicas, clínicas y microbiológicas, para conocer las diferencias con respecto a la tuberculosis en individuos más jóvenes.

\section{MATERIAL Y MÉTODO}

Realizamos un estudio retrospectivo desde 1997 hasta 2002 de todos los casos de tuberculosis con diagnóstico microbiológico confirmado, circunscrito al área sanitaria de Cádiz (España), que comprende las ciudades de Cádiz y San Fernando, con una población estimada de 250.000 habitantes, de los que unos 50.000 son mayores de 65 años, sin apenas variaciones en los años de estudio. Los pacientes procedían de las consultas de atención primaria y del área de urgencias del Hospital Universitario Puerta del Mar de Cádiz.

Durante el período descrito, revisamos las historias clínicas de los pacientes mayores de 65 años diagnosticados de tuberculosis con confirmación microbiológica y recogimos datos referentes a sexo, edad, hospitalización, localización de la enfermedad, clínica, factores de riesgo y antecedentes de tuberculosis. Evaluamos también las características microbiológicas con respecto al resultado de la tinción y el cultivo, así como la sensibilidad de las cepas a tuberculostáticos.

El diagnóstico microbiológico se había realizado en todos los casos por demostración de bacilos ácido-alcohol resistentes mediante tinción de Ziehl-Neelsen o auramina, cultivo en medios de Löwenstein-Jensen y Löwenstein-Jensen con piruvato al 0,4\% (Soria-Melguizo ${ }^{\circledR}$, Madrid, España), e identificación de las cepas por hibridación de ADN con sondas genéticas (GEN PROBE ${ }^{\circledR}$, Bio Mèrieux, Francia) y métodos convencionales ${ }^{12}$. El estudio de sensibilidad de las cepas se realizó por el método de Canetti, Rist y Grosset (SoriaMelguizo ${ }^{\circledR}$, Madrid, España), frente a los siguientes fármacos: isoniazida (INH), estreptomicina (STR), etambutol (ETA), rifampicina (RIF), etionamida (ETI), ácido paraminosalicílico (PAS) y pirazinamida (PZ). Definimos como resistencia global la resistencia a uno o más fármacos antituberculosos, distinguiendo entre resistencia simple y múltiple según afecte a un solo fármaco o a dos o más. Entendemos por multirresistencia la resistencia a isoniazida y rifampicina, independientemente de que pueda incluir también a otros fármacos ${ }^{7-11}$.

\section{RESULTADOS}

En el período estudiado (1997-2002) se diagnosticaron microbiológicamente 302 casos de tuberculosis, de los que $49(16,2 \%)$ correspondieron a pacientes mayores de 65 años. La Tabla 1 refleja la evolución de casos a lo largo de los años, comparando la incidencia en mayores de 65 años con el resto de población. Como se observa, la tasa en población general disminuye gradualmente desde un 26,0/100.000 h en 1997 hasta un $15,2 / 100.000 \mathrm{~h}$ en 2002. Considerando la población mayor de 65 años, la tasa varía bastante de unos años a otros, con una media de 16,3/100.000 h, mientras que la del resto de la población (menores de 65 años) sigue las mismas pautas que la de la población general.

En la Tabla 2, se recogen las características epidemiológicas, clínicas y microbiológicas de los pacientes mayores de 65 años con tuberculosis. El $75,5 \%$ de los casos se registró en pacientes de 
Tabla 1. Evolución de la tuberculosis en pacientes mayores de 65 años en el área sanitaria de Cádiz

\begin{tabular}{|lccccccc|}
\hline Años & 1997 & 1998 & 1999 & 2000 & 2001 & 2002 & Total \\
\hline Número de casos población general & 65 & 61 & 53 & 40 & 45 & 38 & 302 \\
Número de casos mayores de 65 años & 7 & 10 & 5 & 8 & 11 & 8 & 49 \\
Tasa/100.000 hab. mayores de 65 años & 14,0 & 20,0 & 10,0 & 16,0 & 22,0 & 16,0 & 16,3 \\
Tasa/100.000 hab. menores de 65 años & 29,0 & 25,5 & 24,4 & 16,0 & 17,0 & 15,0 & 21,1 \\
Tasa/100.000 hab. población general & 26,0 & 24,4 & 21,2 & 16,0 & 18,0 & 15,2 & 20,1 \\
\hline
\end{tabular}

Tabla 2. Características epidemiológicas, clínicas y microbiológicas de la tuberculosis en pacientes mayores de 65 años

\begin{tabular}{|c|c|c|c|c|}
\hline Características & $\begin{array}{l}\text { Número } \\
<65 \text { años }\end{array}$ & $\begin{array}{c}\text { Porcentaje } \\
<65 \text { años }\end{array}$ & $\begin{array}{l}\text { Número } \\
>65 \text { años }\end{array}$ & $\begin{array}{l}\text { Porcentaje } \\
>65 \text { años }\end{array}$ \\
\hline Sexo masculino & 188 & 74,3 & 37 & 75,5 \\
\hline \multicolumn{5}{|l|}{ Edad } \\
\hline 0-10 años & 23 & 7,6 & & \\
\hline $11-30$ años & 58 & 19,2 & & \\
\hline 31-50 años & 93 & 30,8 & & \\
\hline 51-64 años & 79 & 26,2 & & \\
\hline 65-74 años & & & 27 & 8,9 \\
\hline 75->80 años & & & 22 & 7,3 \\
\hline Hospitalización & 102 & 40,3 & 35 & 71,4 \\
\hline \multicolumn{5}{|l|}{ Sintomatología } \\
\hline Fierre & 75 & 29,6 & 20 & 40,8 \\
\hline Tos & 112 & 44,3 & 22 & 44,9 \\
\hline Hemoptisis & 43 & 17,0 & 5 & 10,2 \\
\hline Disnea & 83 & 32,8 & 22 & 44,9 \\
\hline Síndrome constitucional & 22 & 8,7 & 24 & 49,0 \\
\hline \multicolumn{5}{|l|}{ Factores predisponentes } \\
\hline Antecedentes TBC & 26 & 10,3 & 18 & 36,7 \\
\hline Bronquitis crónica & 16 & 6,3 & 13 & 26,5 \\
\hline Neoplasias & 11 & 4,3 & 5 & 10,2 \\
\hline Diabetes & 17 & 6,7 & 7 & 14,3 \\
\hline Alcoholismo & 53 & 20,9 & 4 & 8,2 \\
\hline ADVP & 42 & 16,6 & 0 & 0,0 \\
\hline VIH & 49 & 19,4 & 0 & 0,0 \\
\hline \multicolumn{5}{|l|}{ Formas clínicas } \\
\hline Pulmonar & 184 & 72,7 & 37 & 75,5 \\
\hline Pleural & 13 & 5,1 & 4 & 8,2 \\
\hline Genitourinaria & 14 & 5,5 & 4 & 8,2 \\
\hline Otras & 42 & 16,6 & 4 & 8,2 \\
\hline Baciloscopia negativa & 89 & 35,2 & 26 & 53,1 \\
\hline Cultivo $<10$ colonias & 38 & 15,0 & 22 & 44,9 \\
\hline \multicolumn{5}{|l|}{ Resistencia fármacos } \\
\hline Resistencia global & 37 & 14,6 & 16 & 32,6 \\
\hline Resistencia simple & 18 & 7,1 & 6 & 12,2 \\
\hline Resistencia múltiple & 14 & 5,5 & 9 & 18,4 \\
\hline Multirresistencia & 5 & 2,0 & 1 & 2,0 \\
\hline
\end{tabular}


sexo masculino; la edad osciló entre 65 y 96 años, con una media de 73 años, siendo el grupo de edad más frecuente el de 65-69 años (34,7\%). En $71,4 \%$ de los pacientes se precisó ingreso hospitalario, 14,3\% procedía de hogares de ancianos, $20,9 \%$ presentaba déficit nutricional y 16,3\% alteración del estado inmunitario.

Los síntomas predominantes en este grupo de población fueron: tos $44,9 \%$, fiebre $40,8 \%$ y disnea $44,9 \%$. Ningún paciente presentó infección por el VIH ni adicción a drogas por vía parenteral; $26,5 \%$ padecía bronquitis crónica y 36,7\% de ellos refirió antecedentes de tuberculosis.

La localización más frecuente de la enfermedad fue la pulmonar $(75,5 \%)$. La afectación radiológica fue unilateral en 15 pacientes (40,5\%). En $16,3 \%$ de los casos la localización fue extrapulmonar (4 genitourinaria, 2 linfática, 1 intestinal y 1 meníngea).

En 11 pacientes con tuberculosis pulmonar fue necesaria la realización de broncoscopia para establecer el diagnóstico (29,8\%). La baciloscopia fue negativa en 26 de las muestras $(53,1 \%)$, incluidas todas las extrapulmonares. El cultivo mostró un crecimiento de menos de 10 colonias en 22 de las muestras (44,9\%).

En el estudio de sensibilidad se detectó resistencia en 16 cepas (32,6\%); de éstas, 12,2\% mostraron resistencia simple: 3 cepas a PZ, 2 a ETI y 1 a RIF, y 18,4\% resistencia múltiple: 2 cepas a INH+RIF, 1 a ETI+RIF, 1 a STR+ETI+PZ, 2 a STR+RIF+PZ y 1 a INH+STR+ETI+PZ. Una de las cepas fue multirresistente, la cual se identificó posteriormente como Mycobacterium bovis de acuerdo con sus características de crecimiento y sensibilidad al TCH.

\section{DisCUSIÓN}

A pesar de la tendencia decreciente de la enfermedad tuberculosa observada en los países desarrollados a finales del siglo pasado, en España continúa siendo un importante problema de salud pública. La tuberculosis en nuestra área sanitaria presenta las mismas características epidemiológicas y clínicas que en otras áreas del país ${ }^{1-3,13-16 . ~}$

Desde la aplicación de un programa de prevención y control de la tuberculosis en nuestra zona a partir del año 1997, observamos una disminución notable de la enfermedad, pasando de una tasa de 26 casos por 100.000 habitantes en dicho año, hasta una tasa de 15,2 casos por 100.000 habitantes en 2002. En el año de inicio de este programa superábamos en cuatro puntos la tasa media de nuestro país (22,2/100.000 hab), mientras que en 2002 (tasa media en España 16,7/ 100.000 hab) nos situamos un poco por debajo ${ }^{2,3}$.

Sin embargo, con respecto a la evolución de la tuberculosis en mayores de 65 años en los últimos seis años, observamos grandes variaciones de unos años a otros y hasta un incremento de la enfermedad en los últimos años, en relación con la población más joven. Esta mayor incidencia de la tuberculosis en ancianos coincide con los datos reflejados en otros trabajos publicados en la Unión Europea ${ }^{17-21}$, aunque en nuestra zona el aparente ascenso puede deberse a la disminución concomitante que se registra en el resto de la población.

Las características epidemiológicas de los pacientes mayores de 65 años siguen las mismas pautas que en la población general en cuanto al sexo, predominando en hombres, con una proporción de 3 a 1 aproximadamente ${ }^{1-3}$. Esto se puede explicar por una mayor prevalencia de factores de riesgo en el sexo masculino, pues se ha demostrado una mayor transmisión de la infección en los hombres por la frecuencia de $\operatorname{contactos}^{22}$. Lo que sí diferencia a los ancianos de la población más joven es el alto porcentaje de pacientes que precisan ingreso hospitalario, debido sin duda a la concurrencia de otras patologías propias de la edad.

La fiebre, tos y disnea son los síntomas predominantes en los pacientes de nuestra serie. Los factores de riesgo difieren de los correspondientes a pacientes más jóvenes; aunque muchos de ellos no pueden considerarse como tales ya que se trata de patologías que se manifiestan especialmente o con más frecuencia en esta edad, como sucede con la bronquitis crónica, las neoplasias y la diabetes. No encontramos ningún caso de infección por VIH ni adicción a drogas por vía parenteral, factores a considerar en la infección tuberculosa en adultos jóvenes. Un factor importante en ancianos es el antecedente de tuberculosis previa a la edad de los 20-40 años, lo que hace 
pensar que en muchos casos se trata de reactivaciones endógenas de infecciones antiguas y no a transmisiones recientes, lo cual ha sido también referido en otros estudios realizados en distintas áreas de nuestro país ${ }^{23,24}$. Este hecho, asociado a factores de riesgo como enfermedades intercurrentes, explicaría una tasa de incidencia mantenida en mayores de 65 años, no afectada por las medidas de control que pueden conseguir disminuir la incidencia en el grupo de menor edad.

En los pacientes mayores de 65 años se diagnostican menos formas de tuberculosis extrapulmonar que en la población joven. Algunos autores han comunicado un alto porcentaje de formas ganglionares y osteoarticulares en ancianos, pero nosotros no detectamos ningún caso de tuberculosis ósea $3,25,26$.

En cuanto al diagnóstico, destaca el alto porcentaje de pacientes diagnosticados mediante broncoscopia y el que la baciloscopia sea negativa en algo más de la mitad de los casos, lo que supone la necesidad de realización del cultivo para establecer el diagnóstico clínico en un elevado número de pacientes. Esta circunstancia condiciona en la práctica la conveniencia de disponer de un microbiólogo experimentado, dedicado al diagnóstico de la tuberculosis, lo que sin duda mejorara la sensibilidad de la tinción como medio diagnóstico. Observamos también que en un considerable número de muestras el cultivo en medio Löwenstein-Jensen presenta escasas colonias, lo cual podría interpretarse como resultado de un proceso infeccioso de

\section{REFERENCIAS}

1. Colnaborative Group for the Study of Tuberculosis IN SPAIN. Epidemiological trends of tuberculosis in Spain from 1988 to 1992. Tubercle Lung Dis 1995; 76: 522-8.

2. De March Ayuela P. Situación actual de la tuberculosis en España. Med Clin (Barc) 1991; 97: 463-72.

3. Boletín epidemiológico semanal. Aislamientos de M tuberculosis notificados al Sistema de Información Microbiológica (SIM) en los años 2000 y 2001. Instituto de Salud Carlos III 2002; 10: 197-200.

4. Vall Mayans M, Maguire A, Miret M, Alcaide J, Parrón I, Casabona J. The spread of AIDS and the re-emergence of tuberculosis in Catalonia, Spain. AIDS 1997; 11: 499-505. baja intensidad, que también podría ser la causa de que la baciloscopia sea negativa ${ }^{27}$. Esta forma de presentación de la enfermedad hace necesario mantener un alto índice de sospecha para lograr un buen diagnóstico.

La resistencia de Mycobacterium tuberculosis a los tuberculostáticos, aunque no es un fenómeno nuevo, es un hecho descrito en diferentes zonas geográficas de España1-3,13-16, Europa ${ }^{17-21}$, América del Norte ${ }^{6,28}$ y del Sur 29,30 y otros continentes ${ }^{31-33}$. En nuestro grupo de ancianos, la resistencia global parece elevada, tanto a favor de la resistencia simple como de la resistencia múltiple. Dado que el tratamiento instaurado con más frecuencia es la triple terapia con rifampicina, isoniazida y pirazinamida, nos preocupa que la resistencia a rifampicina sea especialmente elevada $(12,5 \%)$. La multirresistencia ha desaparecido prácticamente en nuestra área, pasando de 9,2\% en 1997 a ningún caso en 2002; el único caso que encontramos en la población mayor de 65 años fue en 1997, y corresponde a una cepa de Mbovis.

De los resultados de nuestro estudio, podemos concluir que los mayores de 65 años constituyen un grupo en el que incide de forma importante la tuberculosis, con unas características peculiares en cuanto a epidemiología y clínica. Para establecer el diagnóstico microbiológico en estos pacientes se debe recurrir a todas las técnicas disponibles, pues la infección parece se paucibacilar. Además, en nuestra área sanitaria parece conveniente el estudio de sensibilidad para detectar posibles resistencias.

5. Raviglone MC, Snider DE, Kochi A. Global epidemiology of tuberculosis. Morbidity and mortality of a worldwide epidemic. J Am Med Assoc 1995; 273: 220-6.

6. Cantwell MF, Zinder DE, Cauthen GM, Honorato IM. Epidemiology of tuberculosis in the United States, 1985 through 1992. J Am Med Assoc 1994; 272: 535-9.

7. Pérez Fernández JA, Navarro R, Gómez MD. Resistencias iniciales a los principales fármacos antituberculosos en pacientes con infección VIH y tuberculosis pulmonar. Arch Bronconeumol 1993; 29: 93-4.

8. Chaves F, Dronda F, Ortega A, Alonso-Sanz M, LópezCuBero L, GonzÁlez-LóPez et al. Tuberculosis resistente en una población penitenciaria durante el período 1991-1993. Med Clin (Barc) 1995; 105: 81-8. 
9. García-Martos P, González-Moya E, Mira-Gutiérrez J, De la Cale IJ, Pérez-Ramos S, Gutiérrez-RodríGUEz J. Tuberculosis multirresistente en la Bahía de Cádiz. Rev Diagn Biol 2000; 49: 31-8.

10. Ausina V, García-Barceló M, Luquin M. Estudio de las resistencias bacterianas primarias e iniciales en enfermos tuberculosos de un hospital general durante 1983-1987. Enferm Infecc Microbiol Clin 1990; 8: 274-7.

11. CASAl M, GutiérRez J, Ruiz P. Estado actual de la resistencia múltiple a fármacos en la tuberculosis. Rev Esp Quimioterapia 2000; 13: 167-70.

12. Bass JB Jr, Farer LS, Hopewell PC, Jacobs RF, SNider DE. Diagnostic standards and classification of tuberculosis. Am Rev Respir Dis 1990; 142: 725-45.

13. Andrés Puertas C, Mateos Baruque L, Alonso Burgos I, GonzÁlez Megido M. Evolución de la tuberculosis en Palencia. Aten Primaria 2001; 27: 637-41.

14. Campos Rodríguez F, Muñoz Lucena F, Umbría Domínguez S, Reyes NúNez N, De La Cruz Moron Y, Nogaies PÉrEz MC. Evolución de la incidencia de tuberculosis en el área de Sevilla sur (España) durante los 90. Arch Bronconeumol 2002; 38: 221-5.

15. Iglesias Gozalo MJ, Rabanaque Hernández MJ, GomEz López LI. La tuberculosis en la provincia de Zaragoza. Estimación mediante el método de captura-recaptura. Rev Clin Esp 2002; 202: 249-54.

16. PeÑa JM, Ortega A. Estudio transversal multihospitalario de tuberculosis y resistencias en Madrid (octubre de 1993-abril de 1994). Med Clin (Barc) 1996; 106: 1-6.

17. Giove S, Giorgis Ge, ArdizZi A, Barberis S, Bernardi V, GAI R ET AL. Attuale profilo epidemiologico della farmacoresistenza del bacillo di Koch ai chemioantibiotici antitubercolari. Minerva Med 1990; 81: 547-53.

18. Fodor T, VADASZ I. Primary resistence examinations in pulmonary tuberculosis cases in Budapest. Med Thoracalis 1993; 46: 376-81.

19. Gusset T, Rieder HL, Salfinger M. Resistenzhäufigkeit der Tuberkulosebakterien in der Schweiz. Schweiz Rundsch Med Prax 1993; 82: 1101-4.

20. PFyfrer GE. Tuberkulose: diagnostik und Resistenzhäufigkeit 1991/92. Schweiz Rundsch Med Prax 1993; 82: 1090-4.

21. Warburton ARE, Jenkins PA, Waight PA, Watson JM. Drug resistance in initial isolates of Mycobacterium tuberculosis in England and Wales, 19821991. Common Dis Resp CDR Rev 1993; 3: 175-9.
22. Vidal R, Miravitues M, Caylá JA, Torrella M, Martín N, De Gracia J. Estudio del contagio de 3.071 contactos familiares de enfermos con tuberculosis. Med Clin (Barc) 1997; 108: 361-5.

23. Llobet C, Puig T, Falguera M. La tuberculosis en los ancianos. Enferm Infecc Microbiol Clin 2003; 21(Supl 1): 56.

24. Dolz R, Cruselus MJ, Sanjoaquín I, Cuesta J, Letona S, Victoria A ET aL. Tuberculosis en pacientes mayores de 65 años. Enferm Infecc Microbiol Clin 2003; 21(Supl 1): 56-7.

25. Paradisi F, Corti G. Skeletal tuberculosis and other granulomatous infections. Baillieres Best Pract Res Clin Rheumatol 1999; 13: 163-77.

26. Sánchez-Conde M, Ruiz-Serrano MJ, García-Lechuz JM, Marín-Arriaza M, García De Viedma D, Bouza E. Tuberculosis osteoarticular: una entidad infravalorada en el anciano. Enferm Infecc Microbiol Clin 2003; 21 (Supl 1): 43.

27. Godoy P, Nogues A, Alseda M, Manonelies A, Artigues A, García M. Factores de riesgo asociados a pacientes tuberculosos con microscopia del esputo positiva. Gac Sanit 2001; 15: 506-12.

28. McCray E, Weinbaum CM, Braden CR, Onorato iM. The epidemiology of tuberculosis in the United States. Clin Chest Med 1997; 18: 99-113.

29. Valenzueia MT, García P, Ponce J, Lepe R, Veiasco M, PIFFARDI S. Drug resistance of M tuberculosis in Chile: rates of initial resistance for 1986 and acquired resistance for 1985. Bull Int Union Tuberc Lung Dis 1989; 64: 13-4.

30. Pineda-García L, Ferrera A, Gálvez CA, Hoffner SE. Drug-resistant Mycobacterium tuberculosis and atypical mycobacteria isolated from patients with suspected pulmonary tuberculosis in Honduras. Chest 1997; 111: 148-53.

31. Mrtike G, Kebede D, Yeneneh H. HIV infection and antituberculosis drug resistance among pulmonary tuberculosis patients in Harar Tuberculosis Centre, Ethiopia. East Afr Med J 1997; 74: 154-7.

32. Zhang LX, Kan GQ, Tu DH, L JS, Lu XX. Trend of initial drug resistance of tubercle bacilli isolated from new patients with pulmonary tuberculosis and its correlation with the tuberculosis programme in Beijing. Tuberc Lung Dis 1995; 76: 100-3.

33. Curran M, Dawson D, CheaH D. Laboratory surveillance of Mycobacterium tuberculosis isolates in Australia, 1992. Communicable Disease Intelligence 1994; 18: 337-9. 